\title{
CARACTERÍSTICAS CLÍNICAS, DE LABORATORIO Y RADIOLÓGICAS DE PACIENTES PEDIÁTRICOS HOSPITALIZADOS CON COVID-19: SERIE DE CASOS
}

\author{
Héctor Nuñez-Paucar (D1,2,a,b, Jorge L. Candela-Herrera(D)1,3,c Franklin Aranda-Paniora(D1,2,d, \\ Cynthia L. Huby-Muñoz (D)1,4,d, Liz E. De Coll-Vela(D)1,2,d, Raúl R. Bernal-Mancilla(D)1,2,d, \\ Mariela K. Zamudio-Aquise (1) 1,3,a, Sandra C. Schult-Montoya ${ }^{1,2, c}$ \\ 1 Instituto Nacional de Salud del Niño Breña, Lima, Perú. \\ 2 Universidad Nacional Mayor de San Marcos, Lima, Perú. \\ 3 Universidad Peruana Cayetano Heredia, Lima, Perú. \\ ${ }^{4}$ Universidad de San Martín de Porres, Lima, Perú. \\ a Médico/a pediatra neumólogo/a; ${ }^{b}$ magíster en Medicina; ${ }^{c}$ médico pediatra infectólogo; ${ }^{\mathrm{d}}$ médico/a pediatra.
}

\begin{abstract}
RESUMEN
De toda la población infectada por el SARS-CoV-2, la población pediátrica representa del 1 al 5\%, siendo un reto caracterizarla clínicamente. Presentamos cinco casos de pacientes pediátricos con diagnóstico de COVID-19; el rango de edad fue de 1 a 14 años, tuvieron manifestaciones clínicas variadas, tres de ellos presentaron fiebre, tos y dificultad respiratoria, otro fiebre y dermatosis, y un adolescente con diarrea y vómitos asociado al síndrome de Guillain-Barré. Los exámenes de laboratorio revelaron elevación de lactato deshidrogenasa, dimero-D y ferritina. El patrón radiológico más frecuente fue el engrosamiento peribronquial perihiliar. Todos los casos tuvieron evolución clínica y radiológica favorable. La diversidad en las presentaciones clínicas en niños debe considerarse para un diagnóstico temprano de la enfermedad.
\end{abstract}

Palabras clave: Enfermedad por Coronavirus 2019-nCoV; Infección por Coronavirus 2019-nCoV; Pediatría (Fuente: DeCS BIREME).

\section{CLINICAL, LABORATORY AND RADIOLOGICAL CHARACTERISTICS OF PEDIATRIC PATIENTS HOSPITALIZED WITH COVID-19: CASE SERIES}

Citar como: Nuñez-Paucar H, Candela-Herrera JL, ArandaPaniora F, Huby-Munoz CL, De Coll-Vela LE, Bernal-Mancilla RR, et al. Características clínicas, de laboratorio y radiológicas de pacientes pediátricos hospitalizados con COVID-19: serie de casos. Rev Peru Med Exp Salud Pública. 2018;37(4):767-72. doi: https://doi. org/10.17843/rpmesp.2020.374.5296

Correspondencia: Héctor Nuñez Paucar; Avenida Brasil 600, Breña, Lima, Perú; hectornunezpaucar@gmail.com

Recibido: $03 / 06 / 2020$ Aprobado: $23 / 09 / 2020$ En línea: 02/11/2020

\begin{abstract}
Children represent 1 to $5 \%$ of the entire SARS-CoV-2 infected population, and it is challenging to identify them based on clinical characteristics. We present 5 cases of pediatric patients diagnosed with COVID-19; the age range was from 1 to 14 years. They had different clinical characteristics, three of them presented fever, cough and respiratory distress, another one fever and dermatosis, and the other patient had diarrhea and vomiting associated with Guillain-Barre syndrome. Laboratory tests revealed elevated lactate dehydrogenase, D-dimer, and ferritin. The most frequent radiological pattern was perihilar peribronchial thickening. All cases had favorable clinical and radiological evolution. Diverse clinical characteristics should be considered for early diagnosis of COVID-19 in children.
\end{abstract}

Keywords: COVID-19; Coronavirus infection 2019-nCoV; Pediatrics (Source: MeSH NLM).

\section{INTRODUCCIÓN}

La enfermedad por coronavirus 2019 (COVID-19) es una infección causada por un nuevo betacoronavirus, el coronavirus 2 del síndrome respiratorio agudo severo (SARS-CoV-2), que la Organización Mundial de la Salud ha calificado recientemente como pandemia ${ }^{(1)}$.

De toda la población infectada por el SARS-CoV-2, la población pediátrica representa del 1 al 5\% ${ }^{(2)}$. En un estudio retrospectivo en China, de 2135 niños con COVID-19 notificados a los Centros para el Control y la Prevención de Enfermedades chinos, el 34,1\% fueron casos confirmados por laboratorio y la mediana de edad fue de 7 años ${ }^{(3)}$. Otro estudio en Estados 
Unidos demostró que de 149760 casos de COVID-19 solo $2572(1,7 \%)$ de los casos eran menores de 18 años ${ }^{(4)}$.

Las manifestaciones clínicas de la COVID-19 en niños son variadas, la tos y la fiebre son los síntomas más frecuentes ${ }^{(4-7)}$, también se han reportado complicaciones neurológicas diversas ${ }^{(8)}$ y manifestaciones dermatológicas ${ }^{(9)}$, siendo un reto caracterizarlos clínicamente. En niños se estima una tasa de hospitalización del 6 al 20\%, de los cuales $0,6 \%$ a $2 \%$ requieren ingreso a la unidad de cuidados intensivos ${ }^{(4)} \mathrm{y}$ aproximadamente $38 \%$ de estos niños necesitan ventilación mecánica ${ }^{(10)}$. Los casos severos se han asociado a comorbilidades como el asma, enfermedades cardiovasculares, inmunosupresión o enfermedades renales ${ }^{(4,6)}$.

En nuestro medio es importante describir las características clínicas de esta enfermedad en niños, más aún si al 16 de septiembre se registraron 130 pacientes menores de 18 años fallecidos por COVID-19 en el Perú(11).

Nuestro objetivo fue reportar las características clínicas, de laboratorio y radiológicas de la COVID-19 en cinco pacientes de un hospital pediátrico del Perú, enfatizando la variación de presentaciones clínicas.

\section{REPORTE DE CASOS}

Informamos los casos de cinco pacientes que ingresaron al Instituto Nacional de Salud del Niño entre el 2 de abril y el 5 de mayo de 2020. La descripción detallada de hallazgos clínicos, de laboratorio, tratamiento y evolución se presentan en las Tablas 1 y 2.

Los autores hemos obtenido la autorización institucional y los consentimientos informados de los familiares de los pacientes para la publicación de este artículo y de las imágenes que lo acompañan.

CASO 1. Mujer de 1 año. Ingresó a nuestra unidad por fiebre, rinorrea, tos durante seis días y dificultad respiratoria durante dos días. No había tenido contacto con personas con COVID-19 (Tabla 1). El recuento de leucocitos, linfocitos, ferritina y dímero-D fue normal; proteína $\mathrm{C}$ reactiva y lactato deshidrogenasa (DHL) elevadas. La inmunofluorescencia indirecta para adenovirus, influenza (A y B), parainfluenza tipo 1,2 y 3 , y virus sincitial respiratorio fue negativa; la prueba serológica (IgM/IgG) para SARS-CoV-2 fue negativa y la prueba molecular fue positiva (Tabla 2). La

Tabla 1. Descripción de características clínicas, tratamiento y evolución de pacientes con COVID-19

\begin{tabular}{|c|c|c|c|c|c|}
\hline Característica & Caso 1 & Caso 2 & Caso 3 & Caso 4 & Caso 5 \\
\hline Edad (años) & 1 & 1 & 5 & 13 & 14 \\
\hline Género & $\mathrm{F}$ & M & $\mathrm{F}$ & M & M \\
\hline Tipo de exposición & Comunitaria & Comunitaria & Comunitaria & Nosocomial & Comunitaria \\
\hline $\begin{array}{l}\text { Manifestaciones clínicas ini- } \\
\text { ciales }\end{array}$ & $\begin{array}{l}\text { Fiebre, tos, rinorrea, } \\
\text { dificultad respiratoria }\end{array}$ & $\begin{array}{l}\text { Fiebre, tos, dificultad } \\
\text { respiratoria }\end{array}$ & $\begin{array}{l}\text { Fiebre, lesiones } \\
\text { liveloides }\end{array}$ & $\begin{array}{l}\text { Diarrea, vómitos, } \\
\text { debilidad muscular }\end{array}$ & $\begin{array}{l}\text { Fiebre, tos rinorrea, } \\
\text { dificultad respiratoria }\end{array}$ \\
\hline Tiempo de enfermedad (días) & 6 & 2 & 5 & 22 & 6 \\
\hline Fiebre al ingreso & Sí & Sí & No & No & Sí \\
\hline $\begin{array}{l}\text { Pico de temperatura }\left({ }^{\circ} \mathrm{C}\right) \mathrm{du}- \\
\text { rante el curso }\end{array}$ & 38 & 38,5 & 37 & 37 & 39 \\
\hline Enfermedad coexistente & Ninguna & $\begin{array}{l}\text { IDP, acondroplasia } \\
\text { desnutrición crónica }\end{array}$ & Ninguna & Ninguna & Anemia drepanocítica \\
\hline Complicaciones & Neumonía & Neumonía & Vasculitis liveloide & SGB & Neumonía \\
\hline \multicolumn{6}{|l|}{ Tratamiento } \\
\hline Hidroxicloroquina & Sí & Sí & Sí & No & Sí \\
\hline Azitromicina & Sí & Sí & Sí & No & Sí \\
\hline Metilprednisolona & Sí & No & No & No & No \\
\hline Inmunoglobulinas & No & Sí & No & Sí & No \\
\hline Enoxaparina & No & No & Sí & No & No \\
\hline Oxígeno suplementario & Sí & Sí & No & No & Sí \\
\hline $\begin{array}{l}\text { Ventilación mecánica } \\
\text { invasiva }\end{array}$ & No & No & No & No & No \\
\hline Evolución & Favorable & Favorable & Favorable & Favorable & Favorable \\
\hline Estancia hospitalaria (días) & 7 & 10 & 10 & 26 & 5 \\
\hline
\end{tabular}

F: femenino; M: masculino; IDP: inmunodeficiencia primaria; SGB: síndrome de Guillain Barré 
Tabla 2. Hallazgos de laboratorio y microbiológicos de los niños con COVID-19.

\begin{tabular}{|c|c|c|c|c|c|}
\hline Variable & Caso 1 & Caso 2 & Caso 3 & Caso 4 & Caso 5 \\
\hline Leucocitos (VR: $4,5-13,5 \times 10^{3} / \mathrm{mm}^{3}$ ) & 8,64 & 12,26 & 7,32 & 9,64 & 24 \\
\hline Neutrófilos (VR: $1,5-8,0 \times 10^{3} / \mathrm{mm}^{3}$ ) & 3,45 & 7,6 & 1,24 & 5,5 & 18 \\
\hline Linfocitos (VR: $1,5-6,0 \times 10^{3} / \mathrm{mm}^{3}$ ) & 4,8 & 1,59 & 5,2 & 3,6 & 3,6 \\
\hline Plaquetas (VR: $150-350 \times 10^{3} / \mathrm{mm}^{3}$ ) & 158 & 238 & 234 & 187 & 382 \\
\hline PCR (VR: $\leq 0,5 \mathrm{mg} / \mathrm{dL})$ & 1,28 & 2,59 & 0,06 & 0,14 & 5,67 \\
\hline Ferritina (VR: 7 - 140 ng/mL) & 47 & 7 & 750 & 850 & 919 \\
\hline TGP (VR: 0 - 39 U/L) & 20 & 17 & 47 & 55 & 34 \\
\hline TGO (VR: 0 - $47 \mathrm{U} / \mathrm{L}$ ) & 32 & 34 & 87 & 44 & 51 \\
\hline Urea (VR: 10 - 38 mg/dL) & 22 & 13 & 23 & 28 & 21 \\
\hline Creatinina (VR: $0,3-0,7 \mathrm{mg} / \mathrm{dL}$ ) & 0,44 & 0,32 & 0,45 & 0,6 & 0,5 \\
\hline $\mathrm{Na}^{+}(\mathrm{VR}: 135-145 \mathrm{mmol} / \mathrm{L})$ & 140 & 142 & 140 & 142 & 133 \\
\hline $\mathrm{K}^{+}(\mathrm{VR}: 3,5-5 \mathrm{mmol} / \mathrm{L})$ & 4,5 & 4 & 4,4 & 4,6 & 3,6 \\
\hline $\mathrm{Cl}^{-}(\mathrm{VR}: 98$ - $109 \mathrm{mmol} / \mathrm{L})$ & 100 & 107 & 105 & 103 & 96 \\
\hline DHL (VR: 230 - 460 U/L) & 529 & 764 & 1116 & 730 & 1292 \\
\hline CK-MB (VR: 0 - 24 U/L) & - & - & - & 20 & 14 \\
\hline TP (VR: 11,68 - 14,21 s) & 14 & 11,3 & 11 & 12,5 & 12,1 \\
\hline TTPA (VR: 27,12 - 44,21 s) & $>120$ & 14,9 & 24,7 & 18,3 & 34,3 \\
\hline Fibrinógeno (VR: 160 - 369 mg/dL) & 220 & 247 & 228 & 273,8 & 520 \\
\hline Dímero D (VR: <0,5 mg/L) & 0,49 & 2,23 & 1,73 & 0,51 & 5,1 \\
\hline RT-PCR COVID-19 & + & + & - & - & + \\
\hline IgM / IgG COVID-19 & $-1-$ & $-1-$ & $-1+$ & $+/-$ & $-1-$ \\
\hline IFI para virus respiratorios & - & - & NS & NS & NS \\
\hline Hemocultivo & - & - & - & - & - \\
\hline
\end{tabular}

PCR: proteína C reactiva; TGP: alanina aminotransferasa; TGP: aspartato aminotransferasa; DHL: lactato deshidrogenasa; CK MB: creatina quinasa MB; TP: tiempo de protrombina; TTPA: tiempo parcial de tromboplastina activada; RT-PCR: reacción en cadena de la polimerasa en tiempo real; IFI: inmunofluorescencia indirecta; -: negativo; VR: valor referencia.

radiografía de tórax mostró engrosamiento peribronquial perihiliar bilateral. Recibió ceftriaxona, hidroxicloroquina y azitromicina; requirió oxígeno por cánula binasal a $1 \mathrm{~L} / \mathrm{min}$ durante dos días. Tuvo evolución favorable y recibió el alta luego de siete días.

CASO 2. Varón de 1 año. Ingresó a nuestra unidad por fiebre, rinorrea, tos durante dos días y dificultad respiratoria durante un día. No había tenido contacto con personas con COVID-19. Tenía antecedente de inmunodeficiencia primaria (síndrome de hiper IgE) (Tabla 1). El recuento de leucocitos, proteína $\mathrm{C}$ reactiva, DHL y dímero-D fue alto. La inmunofluorescencia indirecta para adenovirus, influenza (A y B), parainfluenza tipo 1, 2 y 3, y virus sincitial respiratorio fue negativa, la prueba serológica (IgM/IgG) para SARS-CoV-2 fue negativa y la prueba molecular fue positiva (Tabla 2). La radiografía de tórax mostró opacidades en vidrio deslustrado bilateral con predominio izquierdo, opacidad alveolar basal derecha y engrosamiento peribronquial hilio basal bilateral
(Figura 1A). Recibió oxígeno por cánula binasal a $3 \mathrm{~L} / \mathrm{min}$ durante tres días y por la condición de inmunodeficiencia se administró meropenem, vancomicina, hidroxicloroquina, azitromicina e inmunoglobulina. Tuvo evolución favorable y recibió el alta luego de diez días.

CASO 3. Mujer de 5 años. Ingresó a emergencia por fiebre y lesiones maculares eritemato-violáceas de patrón reticulado con límites irregulares y mal definidos, no dolorosas, diseminadas en las extremidades inferiores (Figura 1B). No presentaba problemas respiratorios ni había tenido contacto con personas con COVID-19 (Tabla 1). El recuento de leucocitos era normal y el conteo plaquetario también era normal, con agregados plaquetarios. Tuvo valores elevados de DHL, ferritina y dímero-D. La prueba serológica (IgG) para SARS-CoV-2 fue positiva y la prueba molecular negativa (Tabla 2). La radiografía de tórax fue normal. Recibió hidroxicloroquina y azitromicina. Evolucionó favorablemente con alta luego de nueve días. 

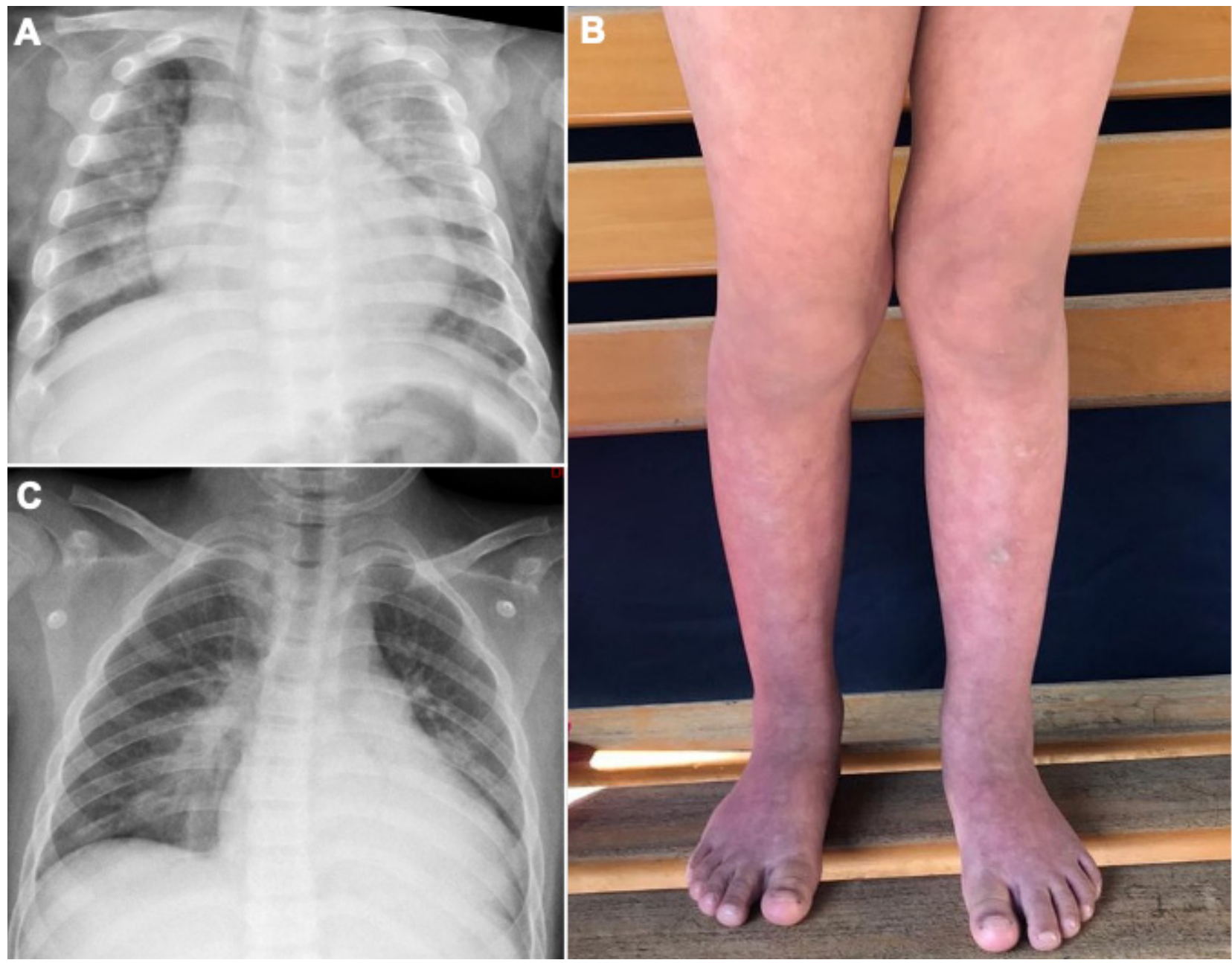

Figura 1. A. Radiografía de tórax anteroposterior de un niño de 1 año con COVID-19 (caso 2): opacidades en vidrio deslustrado bilateral a predominio izquierdo, opacidad alveolar basal derecha y engrosamiento peribronquial hilio basal bilateral. B. Fotografía de una niña de 5 años (caso 3): lesiones con máculas rojizas y violáceas de patrón reticular en miembros inferiores. C. Radiografía de tórax anteroposterior de un adolescente de 14 años con COVID-19 (caso 5): engrosamiento peribronquial hilio basal bilateral y opacidad alveolar basal derecha.

CASO 4. Varón de 13 años. Ingresó a emergencia por vómitos, diarreas, dolor abdominal y debilidad muscular simétrica de comienzo distal y avance proximal, durante cuatro días. No presentaba problemas respiratorios ni había tenido contacto con personas con COVID-19 (Tabla 1). Fue transferido al servicio de neurología; se sospechó de un síndrome de Guillain-Barré, por lo que recibió dos cursos de inmunoglobulina. El recuento de leucocitos, linfocitos y proteína $\mathrm{C}$ reactiva fue normal; ferritina, dímero-D, DHL estuvieron elevados (Tabla 2) y el coprocultivo fue negativo. La prueba serológica (IgM) para SARS-CoV-2 fue positiva y la prueba molecular fue negativa al día 18 de hospitalización. La radiografía de tórax fue normal. Fue transferido a nuestra unidad para aislamiento, no presentó empeoramiento clínico y recibió el alta luego de siete días.

CASO 5. Varón de 14 años. Ingresó a emergencia por dolor abdominal, fiebre, tos, congestión nasal de seis días de evolución y dificultad respiratoria. Tenía antecedente de anemia drepanocítica; no había tenido contacto con personas con COVID-19 (Tabla 1). Presentó hemograma con leucocitosis, hemoglobina 5,7 g/dL; ferritina, DHL, dímero-D y proteína $\mathrm{C}$ reactiva altos. La prueba serológica (IgM/ IgG) para SARS-CoV-2 fue negativa y la prueba molecular fue positiva (Tabla 2). La radiografía de tórax mostró engrosamiento peribronquial hilio basal bilateral y opacidad alveolar basal derecha (Figura 1C). Requirió oxígeno por cánula binasal a $5 \mathrm{~L} / \mathrm{min}$ y transfusión sanguínea. Recibió ceftriaxona, vancomicina, hidroxicloroquina y azitromicina. El paciente mejoró y la anemia drepanocítica se compensó; tuvo el alta luego de ocho días.

\section{DISCUSIÓN}

Los niños infectados con SARS-CoV-2 en su mayoría tienen manifestaciones clínicas leves y se recuperan de una a dos semanas después del inicio de la enfermedad ${ }^{(2,3,5,6)}$. Todos 
los pacientes ingresaron a la Unidad de Contingencia COVID-19 de nuestro hospital, ninguno requirió ingresar a la unidad de cuidados intensivos. En todos los casos se permitió el acompañamiento de un familiar, el cual tuvo serología negativa para SARS-CoV-2, era asintomático y negaba casos sospechosos o confirmados intradomiciliarios.

Los estudios destacan como síntomas principales a la fiebre, en un $44-80 \%$ y tos en un $46-60 \%{ }^{(47)}$. En nuestra serie, el rango de edad fue de 1 a 14 años, tres de ellos (casos 1,2 y 5) tuvieron fiebre, tos y dificultad respiratoria, confirmándose la COVID-19. El caso 3 presentó fiebre que se autolimitó y luego aparecieron lesiones en la piel compatible con el patrón clínico dermatológico liveloide o necrótico, dicho patrón es una de las cinco manifestaciones dermatológicas reportadas por la encuesta nacional de dermatólogos de España y se asocia a un cuadro más severo de la enfermedad ${ }^{(12)}$. Morey-Olivé et al. reportan dos casos dermatológicos en niños con COVID-19 sin compromiso respiratorio ${ }^{(9)}$, que concuerdan con lo observado en nuestro caso. En el caso 4, considerado como síndrome de Guillain-Barré, se desconoce si hay una relación directa entre la debilidad muscular y la infección por SARS-CoV-2. En una revisión sistemática sobre las complicaciones neurológicas secundarias a la infección por SARS-CoV-2 en niños, se observó que de 3707 pacientes el 16,7\% presentó manifestaciones neurológicas inespecíficas (cefalea, mialgia y fatiga) y el 1,13\% manifestaciones neurológicas específicas (encefalopatía, convulsiones y signos meníngeos); y se describieron dos casos de síndrome de Guillain-Barré con respuesta favorable a inmunoglobulinas intravenosas ${ }^{(8)}$.

En nuestros pacientes, el recuento de leucocitos fue normal, excepto en el caso 5. Ningún paciente presentó linfopenia. Los niveles de proteína $\mathrm{C}$ reactiva fueron altos en los casos 1, 2 y 5. Estos hallazgos coinciden con los descritos en otros estudios donde la alteración de los reactantes de fase aguda es menos común en niños, la linfopenia es muy rara y la coagulopatía estaba presente en las formas más graves de la enfermedad ${ }^{(2,10,13)}$. Los casos 3,4 y 5 tuvieron ferritina, DHL y dímero-D altos; el perfil de coagulación fue normal. El caso 3 presentó además agregados plaquetarios y el caso 1 , TTPA prolongado; alteraciones que se han descrito en niños con COVID-19 ${ }^{(10,13)}$.

\section{REFERENCIAS BIBLIOGRÁFICAS}

1. World Health Organization (WHO) (2020). WHO Director-General's opening remarks at the media briefing on COVID-19 - 11 March 2020 [Internet]. WHO; 2020 [citado el 25 de mayo de 2020]. Disponible en: https:/www.who.int/dg/speeches/detail/who-director-general-s-opening-remarks-at-the-media-briefing-on-covid-19---11-march-2020.

2. Ludvigsson JF. Systematic review of COVID-19 in children shows milder cases and a better prognosis than adults. Acta Paediatr. 2020;109(6):1088-95. doi: 10.1111/apa.15270.
Caro-Dominguez $\mathrm{P}$ et al. reportan que de 81 radiografías de tórax de niños con COVID-19, los hallazgos radiológicos anormales más frecuentes fueron engrosamiento peribronquial (58\%) y consolidación (35\%), menos frecuentes las opacidades en vidrio deslustrado (19\%) e infiltrado intersticial (16\%); asimismo, sugieren que la radiografía de tórax sea la primera elección de modalidad de imagen y la tomografía de tórax debe reservarse para casos complejos, complicaciones o posibles diagnósticos diferenciales en niños con comorbilidad ${ }^{(14)}$. En nuestra serie, el caso 1 presentó engrosamiento peribronquial perihiliar; el caso 2, engrosamiento peribronquial hilio basal, vidrio deslustrado y consolidación; el caso 5, engrosamiento peribronquial hilio basal y consolidación. La radiografía de los casos 3 y 4 fue normal. No se realizó tomografía de tórax.

Nuestra serie constituye los primeros casos de COVID-19 en nuestra institución, y al ser casos moderados-graves y con factores de riesgo, recibieron hidroxicloroquina y azitromicina, basados en los documentos de consenso institucional para la fecha ${ }^{(15)}$. El caso 3 recibió, además, enoxaparina, debido a que el patrón liveloide-necrótico podría conducir a oclusión vascular ${ }^{(12)}$. Actualmente no existe evidencia científica que avale la utilidad de azitromicina, hidroxicloroquina, ni anticoagulación profiláctica en el tratamiento de la COVID-19 en niños. Las medidas de soporte general, una adecuada hidratación y el soporte respiratorio en casos críticos son el pilar del tratamiento ${ }^{(10)}$.

Finalmente, la diversidad en las presentaciones clínicas debe considerarse para un diagnóstico temprano de la enfermedad.

Agradecimientos: A los médicos pediatras Raúl A. Rojas-Galarza, Susan V. Genaro-Saldaña, Noé Atamari-Anahui, Jhon D. OrtizPeceros, Edwin A. Miranda-Choque, Werner L. Vásquez-Vidal.

Contribuciones de los autores: HNP, CLHM, LEDCV y RBM concibieron y diseñaron el artículo. HNP, JLCH, FAP, MKZA y SCSM interpretaron los datos e hicieron la revisión crítica del artículo. Todos los autores recolectaron los datos, redactaron el manuscrito, aprobaron su versión final y son responsables de su contenido.

Financiamiento: Autofinanciado.

Conflictos de interés: Los autores declaran no tener conflictos de interés.

3. Dong $\mathrm{Y}, \mathrm{Mo} \mathrm{X}, \mathrm{Hu} \mathrm{Y}$, Qi X, Jiang F, Jiang Z, et al. Epidemiology of COVID-19 Among Children in China. Pediatrics. 2020;145(6):e20200702. doi: 10.1542/peds.2020-0702.

4. Coronavirus Disease 2019 in Children - United States, February 12-April 2, 2020 [Internet]. CDC; 2020 [citado el 25 de mayo de 2020]. Disponible en: https://www.cdc.gov/mmwr/volumes/69/wr/ mm6914e4.htm.

5. Qiu H, Wu J, Hong L, Luo Y, Song Q, Chen D. Clinical and epidemiological features of 36 children with coronavirus disease 2019 
(COVID-19) in Zhejiang, China: an observational cohort study. Lancet Infect Dis. 2020;20(6):689-696. doi: 10.1016/S1473-3099(20)30198-5.

6. Lu X, Zhang L, Du H, Zhang J, Li YY, Qu J, et al. SARS-CoV-2 Infection in Children. N Engl J Med. 2020;382(17):1663-5. doi: 10.1056/ NEJMc2005073.

7. Parri N, Lenge M, Buonsenso D, Coronavirus Infection in Pediatric Emergency Departments (CONFIDENCE) Research Group. Children with Covid-19 in Pediatric Emergency Departments in Italy. N Engl J Med. 2020;383(2):187-190. doi: 10.1056/NEJMc2007617.

8. Panda PK, Sharawat IK, Panda P, Natarajan V, Bhakat R, Dawman L. Neurological Complications of SARS-CoV-2 Infection in Children: A Systematic Review and Meta-Analysis. J Trop Pediatr. 2020;00:1-11. doi: 10.1093/tropej/fmaa070.

9. Morey-Olivé M, Espiau M, Mercadal-Hally M, Lera-Carballo E, García-Patos V. Cutaneous manifestations in the current pandemic of coronavirus infection disease (COVID 2019). An Pediatr (Barc). 2020;92:377-378. doi: 10.1016/j.anpede.2020.04.002.

10. Shane AL, Sato AI, Kao C, Adler-Shohet FC, Vora SB, Auletta JJ, et al. A Pediatric Infectious Diseases Perspective of Severe Acute Respiratory Syndrome Coronavirus 2 (SARS-CoV-2) and Novel Coronavirus Disease 2019 (COVID-19) in Children. J Pediatr Infect Dis Soc. 2020;piaa099. doi: 10.1093/jpids/piaa099.
11. Ministerio de Salud (MINSA). COVID-19 en el Perú - Ministerio de Salud [Internet]. MINSA; 2020 [citado el 16 de setiembre de 2020]. Disponible en: https://covid19.minsa.gob.pe/sala_situacional.asp.

12. Casas CG, Català A, Hernández GC, Rodríguez-Jiménez P, Nieto $\mathrm{DF}$, Lario AR-V, et al. Classification of the cutaneous manifestations of COVID-19: a rapid prospective nationwide consensus study in Spain with 375 cases. Br J Dermatol. 2020;183(1):71-77. doi: 10.1111/ bjd.19163.

13. Henry BM, Lippi G, Plebani M. Laboratory abnormalities in children with novel coronavirus disease 2019. Clin Chem Lab Med. 2020;58(7):1135-1138. doi: 10.1515/cclm-2020-0272.

14. Caro-Dominguez P, Shelmerdine SC, Toso S, Secinaro A, Toma $\mathrm{P}$, Damasio MB, et al. Thoracic imaging of coronavirus disease 2019 (COVID-19) in children: a series of 91 cases. Pediatr Radiol. 2020;50:1354-1368. doi: 10.1007/s00247-020-04747-5.

15. Instituto Nacional de Salud del Niño (INSN). Guía técnica para el diagnóstico y tratamiento de la infección por el nuevo coronavirus (COVID-19) en pediatría [Internet]. INSN; 2020 [citado el 16 de setiembre de 2020]. Disponible en: http://www.insn.gob.pe/sites/default/ files/transparencia/disposiciones-emitidas/GUIA\%20COVID $\% 20$ 19_01-04-2020-095927.pdf. 